\section{A case of terminal ileal prolapse presenting as a polyp}
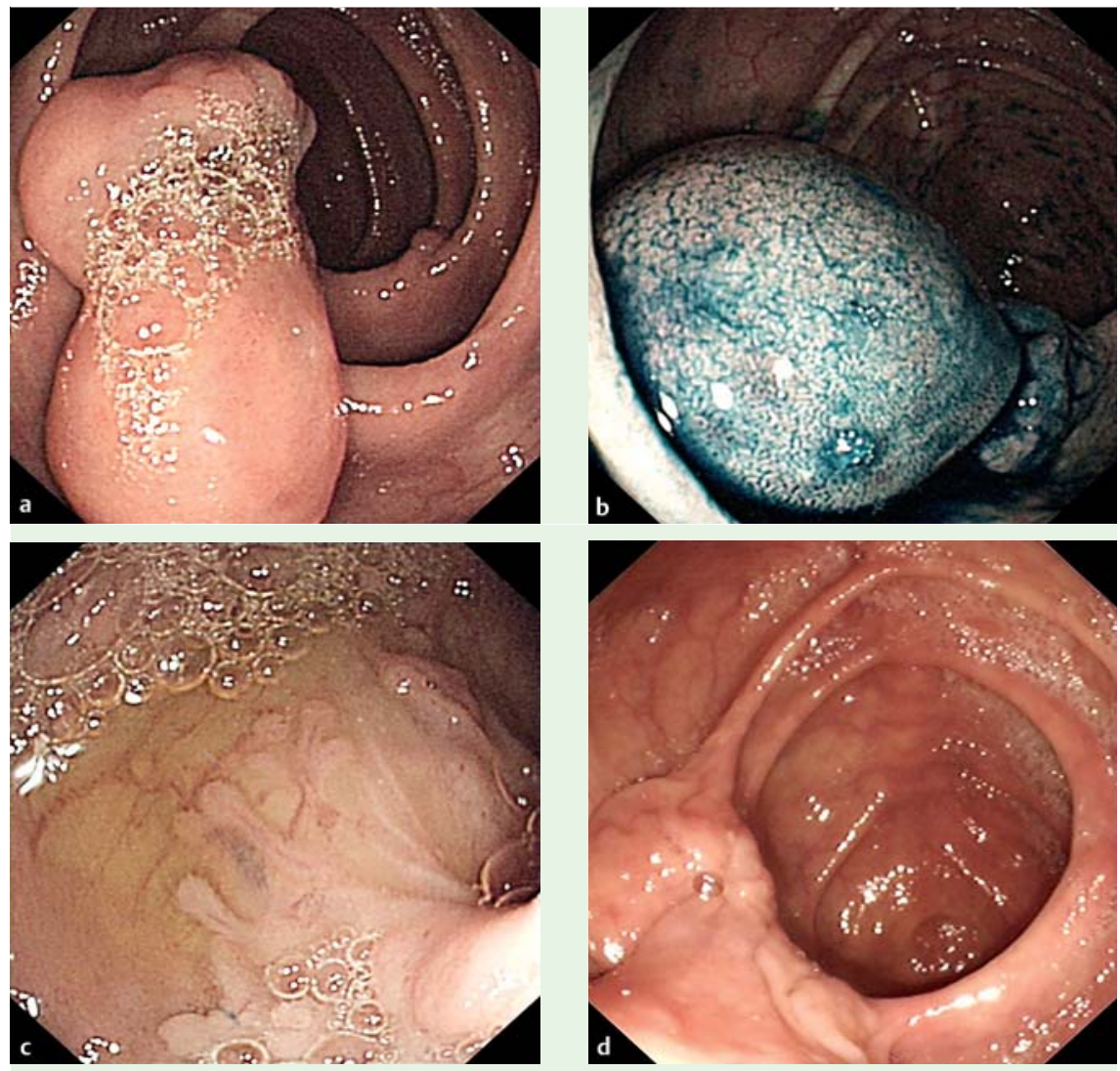

Fig. 1 Endoscopic appearances in a 66-year-old man referred for investigation of persistent diarrhea and iron deficiency showing: a a large polypoid lesion extending from the region of the ileocecal valve; b the surface pattern of the lesion with chromoendoscopy, which demonstrated the appearance of villi; $\mathbf{c}$ the terminal ileum, which was of normal appearance; $\mathbf{d}$ disappearance of the prolapse following ileal intubation.

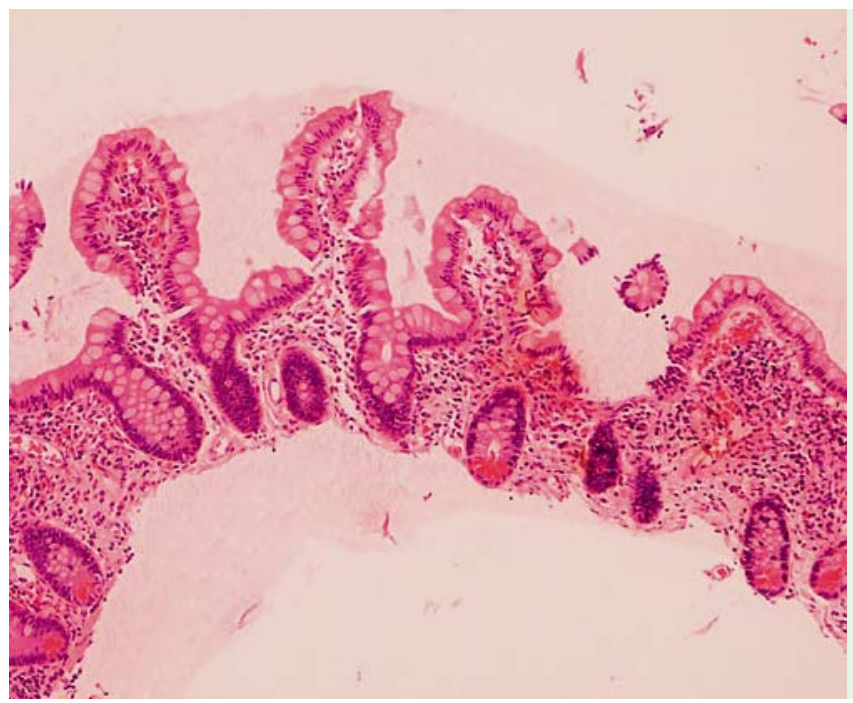

A 66-year-old man was referred for a colonoscopy to investigate persistent diarrhea associated with iron deficiency. A large polypoid lesion $(2 \times 4 \mathrm{~cm})$ was identified at the ileocecal valve ( $\bullet$ Fig. 1 a). Examination with chromoendoscopy ( $0.4 \%$ indigo carmine dye) demonstrated the appearance of villi $($ Fig. 1 b). The terminal ileum had a normal appearance on intubation ( $\bullet$ Fig. 1 c); however, the lesion had disappeared on withdrawal of the colonoscope back into the cecum ( $\mathbf{F i g . 1} \mathbf{1}$ ). Biopsies from the lesion confirmed small-bowel architecture ( Fig.2). This endoscopic appearance in conjunction with the biopsy results was consistent with a prolapsing ileum.

This phenomenon has not previously been reported in the colonoscopy literature. Hawley and Mithoefer reported two cases in 1950 diagnosed on barium studies as a filling defect [1]. In this precolonoscopy era, both patients underwent a laparotomy to exclude malignant pathology; however, only prolapse of the ileum into the cecum was identified. It was suggested that hypertrophy of the ileocecal valve and ileal prolapse often occur together, the prolapse occurring secondary to eversion of the swollen valve.

In our case, the ileocecal valve was not thickened. Hawley and Mithoefer concluded that the presenting symptoms in their patients were not related to ileal prolapse, as they resolved without definitive operative intervention [1]. It is unclear whether prolapse of the terminal ileum is a dysfunctional state that might affect the function of the terminal ileum including bile salt absorption leading to bowel symptoms.

This case highlights the need to carefully assess colonic lesions occurring in the region of the ileocecal valve with modalities such as chromoendoscopy, to prevent misidentification as a polyp and attempted therapeutic intervention.

Endoscopy_UCTN_Code_CCL_1AD_2AJ

Competing interests: None 


\section{K. Keshk ${ }^{1}$, J. Turner ${ }^{1}$, J. Green ${ }^{1}$, M. Morgan ${ }^{2}$}

${ }^{1}$ Department of Gastroenterology, Cardiff and Vale University Health Board, Cardiff, UK

2 Department of Pathology, Cardiff and Vale University Health Board, Cardiff, UK

\section{References}

1 Hawley C, Mithoefer J. Ileal prolapse. Radiology 1950; 54: 380-385

Bibliography

DOI http://dx.doi.org/

10.1055/s-0032-1326496

Endoscopy 2013; 45: E159-E160

(c) Georg Thieme Verlag KG

Stuttgart · New York

ISSN 0013-726X

\section{Corresponding author}

\section{K. Keshk, MD}

Gastroenterology Department Cardiff and Vale University Health Board Penlan Road

Penarth

CF64 2XX

UK

Fax: +44-2920-715538

kkeshk@wales.nhs.uk 